\title{
Téoros
}

Revue de recherche en tourisme

\section{La cuisine régionale traditionnelle...Une cuisine sous influence}

\section{Hélène-Andrée Bizier}

Volume 3, numéro 3, novembre 1984

Cuisine québécoise, restauration et tourisme : vers une synergie

féconde

URI : https://id.erudit.org/iderudit/1080777ar

DOI : https://doi.org/10.7202/1080777ar

Aller au sommaire du numéro

Éditeur(s)

Université du Québec à Montréal

ISSN

0712-8657 (imprimé)

1923-2705 (numérique)

Découvrir la revue

Citer cet article

Bizier, H.-A. (1984). La cuisine régionale traditionnelle...Une cuisine sous

influence. Téoros, 3(3), 20-23. https://doi.org/10.7202/1080777ar d'utilisation que vous pouvez consulter en ligne.

https://apropos.erudit.org/fr/usagers/politique-dutilisation/ 


\title{
...Une cuisine sous influence
}

\author{
par Hélène-Andrée Bizier*
}

On a dit, sans avoir tout a fait tort, que la cuisine québécoise, telle qu'observée au début du siècle était une cuisine morte. empesée dans des méthodes archaïques et peu susceptible d'évoluer. On a tout dit de cette cuisine. On l'a dépréciée tant et plus. au point de forcer la constitution de deux uécolesw: l'une étant résolument contre. l'autre n'ayant en bouche que des adjectifs pour la vanter. Le résultat le plus évident de cette opposition est que l'existence des deux aécoles: est toujours aussi réelle et que l'oeil porté sur la cuisine québécoise traditionnelle est resté imprégné de préjugés, les uns favorables, les autres défavorables.

Quelle est-elle vraiment cette cuisine que ses admirateurs ont modifiée et que ces détracteurs méprisent? Elle est la fille d'une société rurale qui s'est nourrie des éléments fournis par le sol et qui a puisé à travers le gibier, une partie de sa subsistance. Elle est l'enfant d'un peuple souvent frappé par la famine et parfois appauvri par une mauvaise connaissance de son agriculture. La cuisine québécoise, tributaire de l'isolement de certaines régions, s'est permis d'exister en subissant mille et une influences. Influences souvent étrangères, dues au hasard. C'est ainsi qu'il faut accueillir comme un soufflet ou en souriant, l'exemple souvent cité d'un plat que l'on dit typique ude notre cuisine traditionnelles: les très fameuses fèves au lard! $O$ r, la petite histoire des «Boston pork and beansw montre bien que c'est au début du XIXe siècle que ce plat a entrepris son intégration á l'alimentation des Québécois. C'est dans les chantiers oủ travaillent les bûchrons que les propriétaiers de boisés tendent â répandre la recette qu'utilisent les acooks». Bon marché, gras, faisant bon mariage avec les sirops et les sucres qu'affectionnent les habitants de cette partie de l'Amérique du Nord, la recette de fèves au lard pénètre bientôt dans toutes les maisons oủ un bücheron l'a rapportée. A vrai dire, elle n'a jamais vraiment quitté ce monde rural oů. malgré son apparente frugalité, elle a su se rendre indispensable. Mais, de là à préten-

"Hélène-Andrée Bizier est historienne et fut l'une des responsables de la revue Nos Racines.

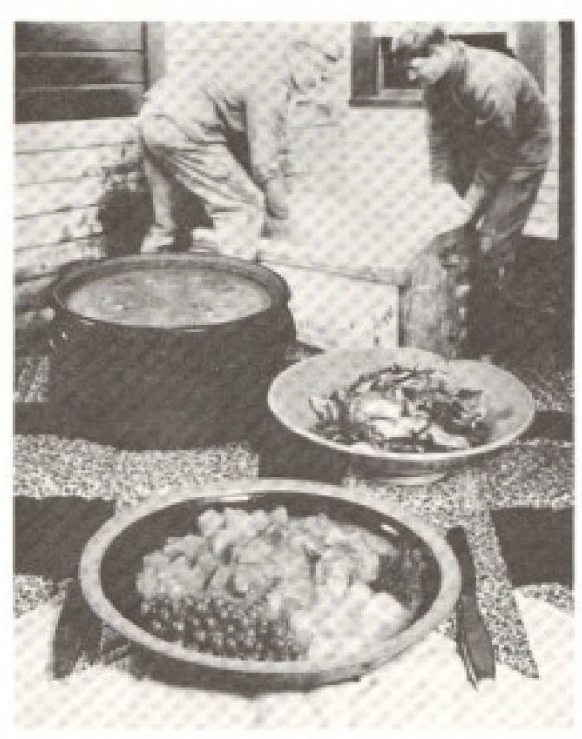

Arrivant en ville, I'habitant transporte avec lui son bagage alimentaire...

dre que ce plat fait partie de l'héritage tra. ditionnel que chaque Québécois porte en lui...

\section{Adieu saveurs et parfums de France}

Le jour mème où un étranger aborda sur les rives du fleuve, il était naturel qu'il emprunte au territoire inconnu les ressources dont il allait avoir besoin pour survivre. Et il le fit. Apress les Cartier, Roberval et Champlain, ce sont des hommes et des femmes tout a fait ordinaires qui tentent de ws'habituer en ce pays nouveau. Les premiers, au XVIle siècle, quittent une province française oủ ils n'ont connu ni l'opulence, ni la sécurité. Pauvreté, chômage. maladie et guerre font partie du menu quotidien. La cuisine régionale française, telle qu'on l'imagine, opulente et savoureuse, est en train de naître mais la gestation s'opère dans des cuisines bourgeoises. Les premiers colons ne la connaissent pas. IIs ne connaissent pas davantage les plaisirs interdits de la chasse et de la pêche. Lorsqu'ils arrivent en Nouvelle-France, leurs bagages contiennent parfois des ustensiles, rangés dans de pauvres coffres, à côté des hardes, des couvertures et de quelques casseroles. En partant, certains emportent dans leurs bagages un parfum de pays. Les biens de la famille Gaspard Boucher sont ceux de nostalgiques craignant de perdre les odeurs et les saveurs familières. "Deux grosses salières à pans, 2 gros violiers ä mettre fleurs, 2 grandes tasses, 6 écuelles, le tout d'étain fin, 8 a dix assiettes, 3 petites écuelles à oreilles, (...) une bouteille de ferre tenant deux pots pleins d'eau de rose, 2 boisseaux de poires cuites, 1 boisseau de prunes aussi cuites..

Et ces parfums, ces saveurs de rose, de poire et de prunes, il les perdent effectivement une fois les provisions épuisées. Les Bretons, les Normands, les Picards, les Beaucerons, les Parisiens et tous les autres, venus d'Auvergne et d'ailleurs, perdent, en émigrant, le contact quotidien avec les produits de leur terroir. Tout est en place pour que débute la chaîne des premiers emprunts conduisant à la naissance de cette cuisine dite québécoise.

\section{D'emprunt en emprunt}

Et voici que des fruits nouveaux s'integrent aux coutumes alimentaires de ces colons qui n'y ont jamais goùtés. La citrouile en est un exemple. Marie de I'Incarnation en est friande et elle voudrait bien que son fils parvienne à en récolter en France. Ele lui en expédie des semences et lui indique quels modes de cuisson sont dejaz en vogue dans la petite colonie: "On les apprete, écrit-elle, en différentes manieres, en potage avec du lait et en friture; on les fait encore cuire au four comme des pommes, ou sous la braise comme des poiresn.

La nature impose sa part de provisions dont la plupart -à l'exception, bien sür, de tous les légumes-racines -sont estimées. Les fruits sauvages poussent partout où I'homme et le feu passent. Les rivières, les lacs et les forêts regorgent d'animaux bons à manger. Certains, comme le castor, l'ours ou la marmotte, osent une incursion dans les casseroles.

L'ancien paysan francais, qui doit louer une vache afin de donner du lait et du beurre a sa famille, n'est pas riche. Sa table ne voit 
pas fleurir les fromages crémeux mais, par contre, ill siapprovisionne aisément en gibier de toute sorte. S'enrichissant par le produit de sa terre, par la vente du bois et par la traite des fourrures, il s'offre un animal dont il raffolait déjà en France, le porc. Après quelques générations passées dans la vallé du Saint-Laurent, I'habitant a également appris â lutterr, à craindre, mais aussi à apprécier l'hiver avec l'assurance de ne manquer de rien. II tire l'eau de la rivière ou du fleuve, par blocs, conserve le lait et la crème congelés.

\section{D'influence en influence}

A la fin du régime français, soit vers 1760 . la cuisine régionale proprement dite n'existe pas car les habitants n'ont pas encore operíe de percée vers l'Outaouais. l'Abitibi, le Saguenay ou vers I'Estrie. Leur cuisine peut être influencée par l'apport de certains éléments particuliers, comme le poulamon rencontré dans la région de Trois-Riviêres, ou comme la gourgane de Charlevoix, mais d'une manière générale, ce qui se mange à Québec se mange aussi à Montréal.

Une certaine cuisine bourgeoise, dépouillée, continue de survivre dans les villes et dans quelques familles, sous I'influence des communautés religieuses féminines. Gardiennes de vieilles traditions, celles que Marius Barbeau a surnommées les csaintes artisanesw, contribuent a former des femmes qui $y$ reçoivent l'éducation complète, telle qu'on la concoit à cette époque. Ces élèves répandent à leur tour certaines techniques, certains secrets reliés autant aux arts de la table qu'd la cuisine. Elles connaissent le secret du gâteau de Savoie, de la confection de l'Angélique, de la préparation de vins doux à base de fruits. Elles distribuent ces connaissances à travers un réseau étroit, plutờt urbain que rural. Les deux modes de vie, celui de la famille bourgeoise citadine et celui de la famille paysanne, ne se rencontrent pas vraiment.

Lorsque survient la defaite des Plaines d'Abraham, la population du pays vit en pleine pénurie alimentaire. L'habitant a été forcé de manger du cheval et des pommes de terre. Sous l'influence des Anglais, qui apprécient ce légume, ils continueront ă en manger. IIs intégreront peu à peu des éléments d'une cuisine étrangère et ils apprendront à cuisiner à la mode de l'Angleterre. Le boeuf et le porc, redevenus abondants, cuiront à longueur de jour, sur le grand poẻle. Cette tendance qui pousse les habitants à tenir, prêtes à être consommees, de pleines chaudrées de légumes et de viande, des miches de pain ventrues, tranchées pour être couvertes de crème et de sucre du pays, leur vaudra cette réputation d'aimer recevoir et donner.

Bien sûr. pour ces gens, les temps de cuisson n'ont aucune importance, pás davantage que la longueur des sauces, ou que la qualité des fonds. Ces thèmes ne les inté:ressent pas. Ce qui compte, cest de manger quand, par bonheur, on peut le faire.

\section{À la recherche de nouvelles avenues}

Au XIXe siècle, le pays nourrit mal ses habitants. La terre ne rend plus prospère. Elle ne produit pas de riches. La vie de I'habitant, au contact du progrès, perd une partie de son charme. Loin des villes, on idealise l'existence des citadins. On veut profiter de l'argent qui y coule et, pour cela, on quitte la campagne oủ sans trop en réaliser l'importance, on était propriétaire. On quitte la campagne pour les Etats-Unis ou pour Montréal où les manufactures dévorent les santés.

Arrivant en ville, I'habitant transporte avec lui son bagage alimentaire. Les fẻves au lard, la soupe au pois, nourritures de trappeurs et de bücherons, viennent prendre la place du boeuf et du porc, désormais trop chers. La farine de sarrasin remplace souvent le blé. Trop cher. Pour se nourrir comme il le faisait chez lui, le nouveau venu attendra Noël ou la richesse promise!

Ce scénario alimentaire se répète aussi pour I'habitant de la vallée du Saint-Laurent que son amour de la terre pousse vers les terres à défricher: Estrie, Beauce, Saguenay, Outaouais. Deux cents ans après la venue des colons français, ils recommencent, ailleurs, sur des sols moins fertiles, dans des régions oủ ils s'isolent malgré eux. A leur tour, ces pionniers sont forces de tirer les ressources alimentaires de leur environnement. Ils découvrent la ouananiche, transplantent la gourgane, s'intéressent aux champignons, apprivoisent le canard, développent les produits de l'erable en créant ce qui s'appelle aujourd'hui une cuisine régionale traditionnelle.

\section{La pire des influences}

Le XXe siècle arrive. De plus en plus de gens ont woyagé et de nombreuses personnes, les médecins en tête, s'intéressent à I'alimentation de leurs compatriotes, Ils lui trouvent déjả mille défauts. Mais ils parlent, et c'est cela qu'il faut retenir, de cette cuisine transportée en ville et dans les villages par d'anciens habitants qui n'ont pas modifié cet aspect de leur existence. Ils éveillent, et avec raison, l'attention des gens sur les dangers de se nourrir d'aliments gras et indigestes qui n'ont plus leur place dans l'alimentation quotidienne des ouvriers.

Mais quoi faire? Quoi changer lorsqu'on est encore trop pauvre pour modifier son régime alimentaire?

Le message, cent fois répété par les médecins et par les gastronomes qui appartiennent aux premières sociétés qui naissent au début du siècle, finira par étre compris. Peut-être mal compris.
L'arrivée massive des conserves alimentaires, la mise en marché des pâtes, et des élements tout faits, sauces a diluer, soupes à mouiller, etc., vont provoquer une réaction inattendue, qui s'observe encore. Les ménagères, puisque c'est ainsi qu'on désignait alors les consommatrices, consentent à moderniser leur garde-manger. La soupe aux pois et les fèves au lard deviendront des mets d'exception. Comme pour les tourtières et les cipâtes qu'on mangera surtout à l'occasion des fểtes.

Puis, comme si le message avait été mal compris, la période survenant après la deuxieme grande guerre est celle oú apparaissent les succédanés qu'on adopte avec plaisir... Et, c'est là qu'on assiste â la disparition de la cuisine qu'une tradition terrienne avait transportée en ville. De temps à autre, on souhaite la faire revivre. Mais d'après quel modèle le fera-t-on? D'après celui de la cuisine bourgeoise, survivant grâce aux religieuses? D'après celui de la cuisine régionale?

N'est-il pas temps de se retourner du côté du terroir, d'assimiler les odeurs et les saveurs de celui-ci et de mettre en valeur les caractéristiques propres à notre sol dont les produits sont parfois plus doux, plus abondants et quelquefois, malgré tout, plus amers qu'ailleurs...?

\section{Bibliographie}

SULTE, Benjamin, Histoire des Canadiens Français. 1882, 1884

BOUCHER, Pierre (1684I. Histoire váritable et naturelle des moeurs des productions du Pays de la Nouvelle-France vulgairement dite le Canada, Societé historique de Boucherville, 1964.

SEGUIN, Robert Lionel La civilisation traditionnelle de I'uhabitants au XVIle at XVIlle siècles, edition Fides, 1973.

DOUVILLE, Raymond et CASANOVA, Jacques $D_{1}$, La vie quotidienne en Nouvello-France, edition Hachette 1964, nouvelle dedition 1992, LRP, Montréal,

LAcoufsiefe, Jacques et BIzIER, Mạrie-Helêne, Nos Racines, I'histoine vivante des Quebbecois, fascicules 15, 28, 73, ddition Transmo, 1979, 1981 .

ROUSSEAU, Jacques et BETHUME, Guy. Voyages de Pehr Kalm au Canada en 1749. traduction anno. tée du journal de route, Le Cercle du Livre de France. 1977

LAVERDIERE, C.-H., Oeuvres de Champlain, présentées par Georges-Emile Giguere, éditions du Jour, 1973. 
Le poivre, la muscade et toutes les épices orientales se vendaient á prix d'or et, pour acheter les services d'un fonctionnaire ou pour régler une note un peu salée, quelques grains de poivre valaient souvent un pot de vin.

Le cours des épices était tel, leur faveur si remarquable, qu'un jour les rois décidêrent de conquérir le monde pour s'en approvisionner. Et c'est parce qu'ils recherchaient des épices autant que de l'or que les les explorateurs enteprirent de sillonner les mers. C'est à cause de cette longue quête de parfums nouveaux, de fruits nouveaux, cachés dans de lointains pays, que le hasard conduisit ces aventuriers vers le littoral américain.

Croyant naviguer vers I'Inde en empruntant une route maritime nouvelle qui le conduit vers l'Est, Christophe Colomb commet une heureuse erreur dont il ne mesurera peutêtre jamais l'importance. Sur le plan strictement alimentaire, il découvre le mails. Croyant être aux Indes, il donne à ce blé le nom de ablé d'lindew. II fera la mẻme chose pour le coq et le ecochon d'Inder. Les spécimens qu'il rapporte en Espagne seront introduits dans les autres pays européens d'où ils reviendront plus tard, anoblis par un usage régulier dans la cuisine des pionniers. Selon le botaniste et ethnologue Jacques. Rousseau, elles sont pres de 80 , les plantes alimentaires qui se sont intégrées à la cuisine européenne depuis Colomb. Parmi celles-ci, on trouve le manioc, I'arachide, la pomme de terre, la tomate, le piment, la citrouille, etc.

\section{Un monde mésestimé}

L'Amérique était riche, mais le hasard a voulu qu'on ne lui attribue pas spontanément le merite qui lui revenait au chapitre alimentaire.

A la même époque, peut-être, les pêcheurs basques fréquentaient les bancs de TerreNeuve et les eaux du golfe Saint-Laurent à la recherche d'une denrée essentielle à l'Europe catholique: la morue. Jeûne et abstinence, de concert, imposaient les menus dits maigres, un jour sur deux ou presque. Les pêcheurs européens qui s'aventuraient sur le fleuve Saint-Laurent multipliaient leurs sources de revenus en traitant la chair et l'huile de baleine.

Lorsqu'arrive Jacques Cartier en 1534, les pécheurs basques sont encore installés à l'entrée du pays et si Jacques Cartier pénètre enfin dans le golfe, en 1535 , c'est dans l'espoir de trouver, grâce à ces cours d'eau, la route menant à la Chine. Le vieux rêve de découvrir or et épices est plus vivace que jamais.
Mais Jacques Cartier n'est pas un visiteur, ou un touriste blasé. Tout lui est sujet d'émerveillement et on peut se féliciter de l'existence de son récit de voyages. Pour lui-même comme pour les membres de la première expédition qui le conduit sur les rivages du apays de Canadaw, apres la terre et le paysage, c'est le fait de s'alimenter qui importe. On comprend cette prétoccupation lorsqu'on sait que les provisions de bouches, lors des traversées, sont soumises aux ravages de l'humidité, que l'eau se corrompt et que les aliments cuits ne font pas partie du menu quotidien. Lorsqu'ils crient aterrew, les marins de Cartier savent déjà qu'ils vont trouver de quoi satisfaire leur appétit. Le 21 mai 1534 , au terme d'une traversée ayant duré moins de 20 jours, ils quittent le navire à bord de deux chaloupes pour chasser. Ce témoignage de Cartier est l'un des premiers à évoquer toute l'abondance que l'on devine à l'intérieur d'un continent qui se révélera être aussi giboyeux qu'il paraissait l'être. Les hommes vont vers l'île à laquelle Cartier donne le nom d'ile aux Oiseaux parce que justement, les oiseaux sont "grands comme des oies, noirs et blancs, et ont un bec comme un corbeaun. Le régime des bis. cuits de marin vient de prendre fin. Lui succède l'abondance puisque, aen moins d'une demi-heure, nos deux barques en chargerent comme des pierres.n On les mangera frais, mais également salés puisque c'est le mode de conservation le plus commun: aChacun de nos navires en sala quatre ou cinq pipes, sans compter ce que nous avons mange de frais?.

Et voici qu'après le gibier à plumes, le gibier a poil s'offre a la convoitise des voyageurs. Le golfe Saint-Laurent vient d'y gagner la réputation qu'il conserve, quatre cents ans après le voyage du Maloin. «Faisant route vers terre, hous trouvames /"ours à environ la mi-chemin, qui allait aे terre aussi vite que nous le faisions à voile. L "ayant apercu, nous lui donnämes la chasse avec nos barques et nous le primes de force. Sa chair était aussi bonne que celle d'une génisse de deux ans. $\|_{+}$

\section{Le premier itinéraire gastronomique}

Les voyages subséquents de Jacques Cartier lui permettront de dresser, à son insu sans doute, un très intéressant itinéraire gastronomique. Aux Iiles-de-la-Madeleine, il est séduit par le ablé sauvagew et les upois en fleurs\%. Les fruits sauvages que l'on cueille encore avec autant de plaisir, sont au rendez-vous, ainsi que les fines herbes. L'ile de Brion voit aussi pousser les roses de Provins dont la médecine balbutiante fait un généreux usage. A l'état pur, à l'état sauvage, le opays de Canadas plaît déjà à Cartier qui, souhaitant malgré cela trouver la route de l'Orient, pénètre plus avant dans le continent.

La tournée agastronomiquen de ce touriste pionnier se poursuit en 1535, à travers les anguilles offertes par les Amérindiens, le mil dont les premiers habitants tirent une espece de pain, les fruits sauvages encore et de gros melons. Du côté de Québec, à I'ille d'Orléans, il voit des vignes portant de beaux raisins qui pourraient être cultivés. L'île recevra d'abord le nom d'īle de Bacchus. Vers Montréal, Cartier est initié à une cuisine locale dépourvue de sel: potages, poissons, pains et haricots apprêtés à une mode qui ne leur convient pas: "Parce que ces vivres n'étaient pas à notre goüt et qu'il n'y avait aucune saveur de sel, nous les remerciames en leur faisant signe que nous n'avions pas besoin de manger.. Si Jacques Cartier peut refuser cette invitation et d'autres, c'est sans doute parce que la nature leur offre encore de quoi se nourrir. A bord de l'une des deux barques qui ont remonté le fleuve depuis le lac Saint-Pierre, se trouvent peut-être une ou deux upipesn remplies à ras bord de poisson ou de viande. A moins qu'empruntant aux Amérindiens cette simplicité si imitée depuis, ils n'aient mangé des aliments frais, cuits sur les pierres du feu ả même le sol.

Quoi qu'il en soit, Cartier et ses hommes sont à la vieille de ne plus pouvoir refuser ce qu'on leur offre. Dans quelques semaines, ils vont rencontrer le plus cruel ennemi de l'homme du Nord: le froid. Le plus solide allié aussi. Car s'il peut tuer, cet élément permet une meilleure conservation des aliments. Mais cela, Cartier n'aura pas le loisir de l'analyser car pour lui, le froid signifiera d'abord isolement, puis maladie. Au cours de I'hiver 1535, les aliments gèlent, ainsi que le cidre et le vin, dans un navire oú le froid construit un mur de glace de uquatre doigts d'épaisseurs.

C'est aux Amérindiens que Cartier empruntera le secret lui permettant de soigner ce qui reste d'un équipage que le scorbut lui dispute. C'est la premiere fois qu'il est question d'une infusion miracle à base d'annedda. L'écorce de cèdre blanc fait merveille. Les hommes de Cartier que la maladie avait dẹjâ touchés se relèvent guéris.

\section{Pour apprivoiser ce pays}

II faut attendre une soixantaine d'années après les voyages de Cartier et celui de Roberval qui ont conduit à une infructueuse tentative d'établissement permanent pour revoir des explorateurs observer les rives du fleuves. 
Samuel de Champlain recherche lui aussi la route conduisant à l'Ouest, aux épices et â l'or. Sur la route des épices, il rencontre Québc dont il ne se détachera pas. Pour lui aussi, tout commence par l'estomac. Son regard embrasse d'abord le paysage. II construit une habitation qui, avant toute chose, devra protéger un potager.

Les relations avec les autochtones permet= tront aux Français de mieux connaitre les ressources du territoire et de l'apprécier tel qu'il est. L'expérience de Jacques Cartier avec le scorbut a été utile aux Français qui I'ont suivi en Amérique. C'est à Port-Royal que Samuel de Champlain avait expérimenté l'Ordre de Bon Temps en 1606-1607. II la rếpète â Québec en 1608, obligeant ses compagnons à accepter, chacun à son tour, la tåche de chasser ou de pécher pour le bénéfice commun. C'est alors que le pays se révèle. L'hiver suivant sera pour les hommes de Champlain l'un des plus agréables qui soient puisque wle p/us grand de leur travail était de se donner du bon tempss.

Dix ans après son arrivée ả Québec, Champlain s'estime heureux. Les vignes qu'il a plantées ont donné leurs fruits. La terre qu'ill a désertée (rendue sesmblable à un désert), labourée et ensemencée a porté tous les fruits espérés. Désormais, on pourra vivre sur ces terres: aJe $n$ 'avais d'autre dessein, écrit-il, en 1618, que de voir le pays peuplé de gens laborieux, pour défricher les terres, afin de ne point $s$ 'assujettir a porter des vivres annuellement de Frances.

\section{L'autosuffisance}

Samuel de Champlain l'avait compris: Québec et la vallée du Saint-Laurent ne pourraient pas être exclusivement des comptoirs de traite. Les habitants ne pourraient pas compter sur des provisions transportées par mer. Ils devraient viser l'autosuffisance, au moins au chapitre de l'alimentation.

Les réserves de gibier semblaient inépuisables et procuraient aux premiers habitants des plaisirs que nous imaginons mal. Ainsi, Robert Giffard découvre et apprécie les plaisirs de la chasse. Alors que Québec est encore une étroite bourgade, Giffard apprécie la solitude d'une cabane oủ il se rend pour traquer le canard. La Canardière lui doit son nom. Les conditions de vie quotidienne qu'on croit difficiles l'étaient sans doute, mais pour des gens qui avaient connu les privations d'un pays presque continuellement aux prises avec la guerre, la famine ou l'épidémie, les rives du Saint-
Laurent ont pu sembler paradisiaques. On voit les colons se réserver le droit de pêcher l'anguille et le saumon. Transformer le gibier aquatique, les oiseaux de basse-cour en monnaie d'échange. On les voit cultiver, longtemps avec des instruments aratoires en bois, sans secours animal, des terres dont la superficie augmente, bon an, mal an, d'un arpent par homme au travail.

Et, pour ces héritiers de Jacques Cartier et de Champlain, I'autosuffisance devient un objectif. On continuera d'importer le sucre, les écorces d'orange et de citron, le sel, le poivre et les quatre épices dont on abusera... comme en France. Mais on tirera profit des saveurs et des parfums nouveaux. II ne leur sera plus possible de reproduire des modeles culinaires identifiables aux régions d'oủ ils viennent. Dans un pays oủ anguilles et saumons abondent, où le froid de l'hiver facilite la conservation de toutes les denrées, y compris celle de la viande de porc, on crée une cuisine adapthe au climat et aux ressources du territoire.

C'est une cuisine dépouillée d'artifice. réduite à sa plus simple expression qui naît, au XVlle siècle, de l'éloignement de la province natale et de l'obligation de ne comp ter que sur soi. $f$

\section{(H.-A. Bizier)}

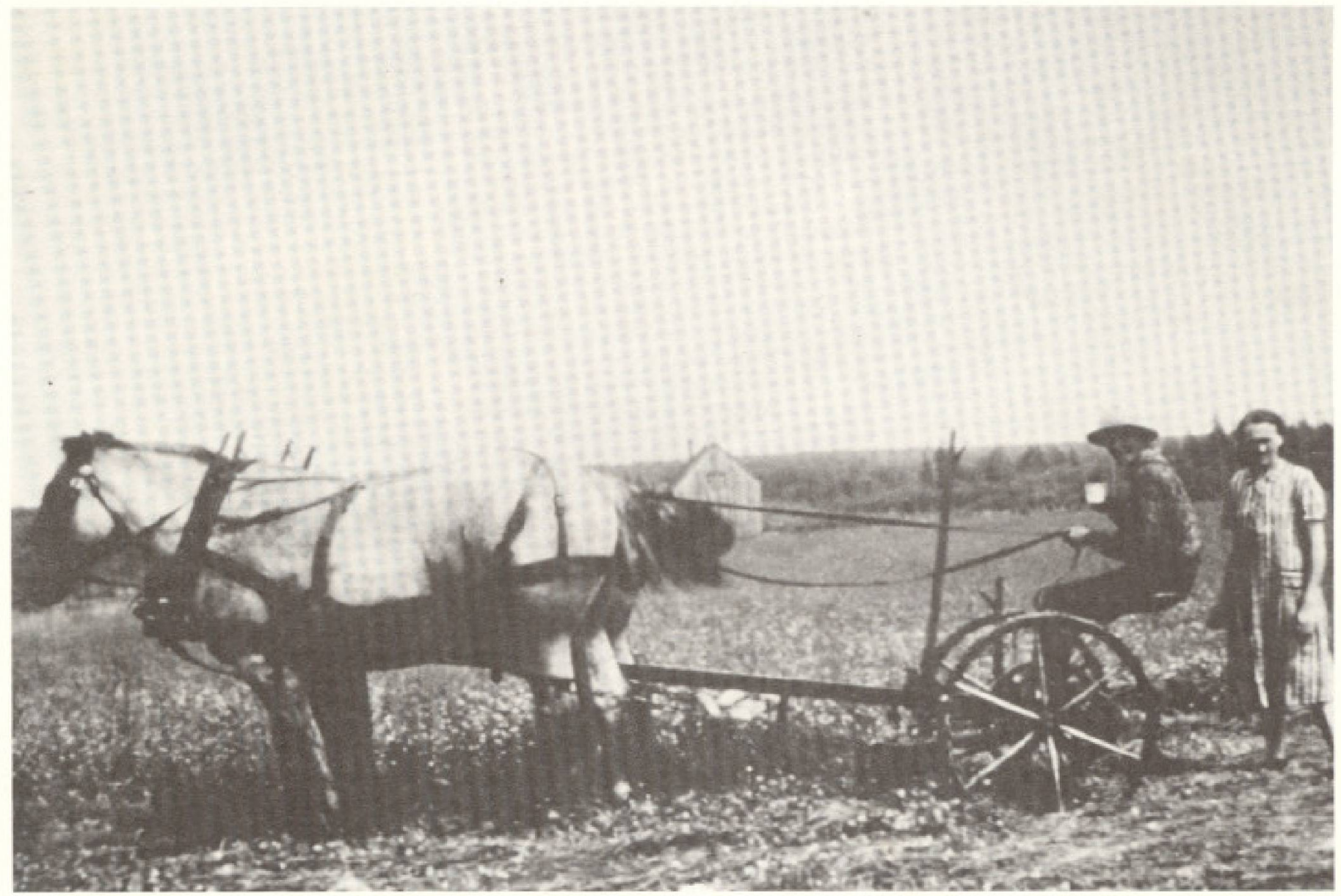

Deux cents ans après la venue des colons français, ils recommencent ailleurs sur des sols moins fertiles... ces pionniers sont forcés de tirer les ressources alimentaires de leur environnement. 